\title{
DEFORESTATION DUE TO URBANIZATION: A CASE STUDY FOR TRABZON, TURKEY
}

\author{
C. Telkenaroglu a, M. Dikmen ${ }^{\mathrm{a}, *}$ \\ ${ }^{a}$ Baskent University, Computer Engineering Faculty, 06790 Etimesgut Ankara, Turkey - cantelk@gmail.com, \\ mdikmen@ baskent.edu.tr
}

KEY WORDS: Deforestation, Landsat 7 ETM+, Vegetation Index, Ground-Truth, fScore Statistics

\begin{abstract}
:
This paper inspects the deforestation of Trabzon in Turkey, due to urbanization, between 2006 and 2016. For this purpose, Landsat 7 ETM+ (Enhanced Thematic Mapper Plus) images are obtained from United States Geographical Survey (USGS) archive (USGS, 2017a) and their VNIR bands related to this study are utilized. For both years, and for each band, histograms are equalized. Finally, Normalized Difference Vegetation Index (NDVI) values are calculated as images. Resulting vegetation indexes are assessed in comparison to the binary ground truth images. A visual inspection is also done with respect to Google's Timelapse images for each year to validate and support the results.
\end{abstract}

\section{INTRODUCTION}

Deforestation, due to fires and urbanization is a major issue today. Turkey's black sea region is very rich in vegetation. Because of warm winter temperature, nuts, tea barley, wheat, rye, lentil, cotton, corn, apple, beet, linen, hemp, soybean, olive, citrus, kiwi, tobacco are easily cultivated. $\% 70$ of the population live on agriculture. Trabzon and its neighbors (i.e., Giresun and Ordu) produce $\% 85$ of all the nuts produced in Turkey and along the shores of Rize \%100 of Turkey's tea is produced (MSXLAB Forum, 2008). Besides being very flourished, Black Sea also is world's first exporter of nuts.

However, Black Sea region is also very popular for tourism any season, especially during summers, with its plain, cool and green areas. This area also contains cities, which keep on growing. Since the land is very productive, population prefers to stay instead of migrating to other cities and this ends with further urbanization. This study inspects Trabzon' s change of vegetation between 2006 and 2016 due to this urbanization using Landsat 7 Imagery.

\section{BACKGROUND}

During last decades, deforestation has been studied both locally or globally. Use of satellite images has made the studies more accurate and precise. Various approaches have been followed using satellite images. Most studies have chosen to classify the images. For instance, Pacheco et al. used the MODIS satellite imagery from 2005 to 2010 , and picked random samples to train a decision tree model to classify regions to detect deforestation hotspots in Venezuela (Pacheco, 2014). They verify their results using Google Earth images and expert opinions. Similarly, Menaka et al. performed polygon segmentation to split forest and urbanized areas, however they perform 2D Haar Wavelet Transformation with different thresholds and at a low rate they clear out the noise such as clouds or shadows (Meneka, 2013). Machado et al. implemented an image segmentation and classification neural network model, which is trained by backpropagation to analyze deforestation in Amazon rainforests (Machado, 1993). For high resolution satellite images, segmentation performance may become a problem due to the increase in detail. In a different study, Csillik used Random Forest classification on overly segmented data. This work utilized Simple Linear Iterative Clustering (SLIC) of superpixels, which reduce the amount of time needed for partitioning (Csillik, 2017).

In order to study satellite images properly, noises and distortions must be dealt with first. Hollstein et al. inspected existing algorithms like decision tree, bayesian model, random forest, which are found potentially successful in partitioning clouds, cirrus, snow, shadows and clear sky areas in Sentinel satellite imagery of $10 \mathrm{~m}$ resolution (Hollstein, 2016). Besides noise detection, another raw satellite image pre-processing step is to fill the missing scan lines. For example, SLC (Scan Line Corrector), which compensates the forward movement of the satellite, is a small mirror in optical path of the Enhanced Thematic Mapper (ETM+) instrument, has failed on May 31, 2003. Resulting large number of missing scan line patterns in al the imagery of Landsat 7. Studies state that the histogram equalization or missing line fixing techniques prefered by USGS (USGS, 2017b, 2017c) has limitations against heterogeneous scenes, instead kriging, or geostatistical techniques (Zhang, 2007, McCabe 2017) have more satisfactory results.

The rest of the paper is organized as follows; Next section describes the approach. The 4th section concludes the paper.

\section{APPROACH}

For this study, we have used Landsat 7 ETM_ C1 Level-1 satellite imagery archive to gather data with maximum $\% 20$ cloud cover. Possible alternatives were Landsat 8 images, which is free of missing lines or Sentinel $1 \& 2$ satellites which has a lot higher spatial resolution (10 meters). The reason they were not chosen is that, they were launched very recently, in 2014, which will not allow for inspection in a longer time period.

Coordinates in surrounding Trabzon were picked to query satellite images in USGS webpage. As a result, the study area covering Trabzon was chosen as illustrated in Figure 1.

\footnotetext{
* Corresponding author
} 


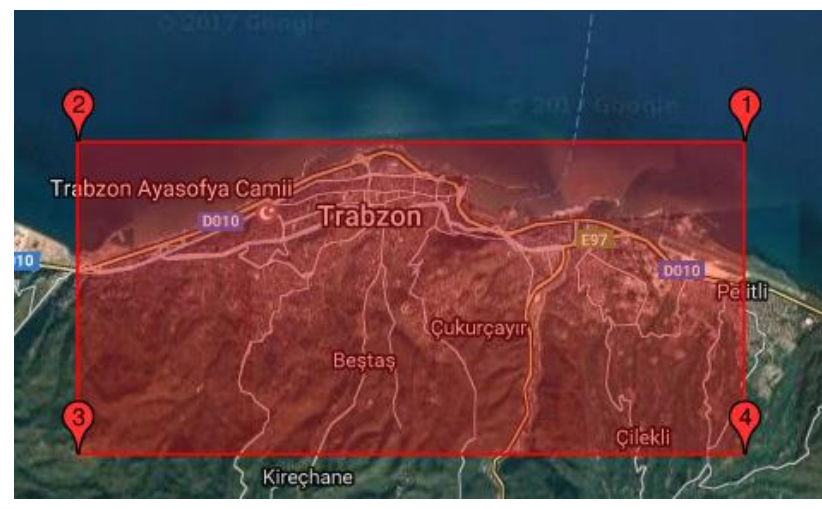

Figure 1. The study area covering Trabzon

Further, images between year 2000 and 2016 were queried for months of summer season. Winter, spring and fall season are not preferred because of heavy snow or cloud cover in the region, and such regions usually have a very low negative NDVI value (FSNAU, 2017), which makes it difficult to discriminate forestry from other areas. In addition, during warm months, it is more likely that cloud cover will be minimal.

The approach is implemented using Matlab R2017a. All band images were rotated 11 degrees with cubic interpolation. Then, the region of interest was cropped resulting a $1000 \times 350$ pixel image. Missing lines in the satellite images are removed using the gray-level morphological closing operation (MathWorks, 2017). MatLAB, is able to performs this operation on multichannel color images, as they are treated as volumetric images such as MR or CT, once a structuring element that lies in a $2 \mathrm{D}$ geometry is used. We have used a thick line (6 pixels in height and 50 pixels in length) as a structuring element. Resultant image is shown in Figure 2.

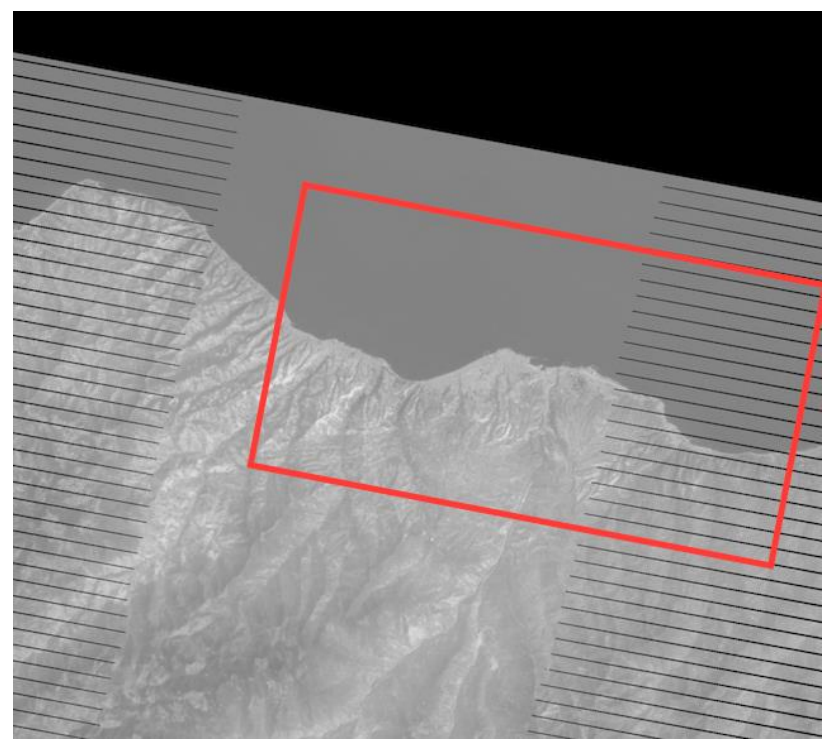

Figure 2. Missing lines in the satellite image of the region of interest

Landsat 7 has eight bands: blue, green, red, near-infrared 1, near-infrared 2, thermal, mid-infrared, panchromatic. All of the bands have 30 meters resolution, except thermal with 60 and panchromatic with 15 meters of resolution (GIS Geography, 2017, USGS, 2017). Picked bands of the imagery are band 2 , band 3 and band 4, representing, green, red and near infrared bands. These bands are selected since they are used in the calculation of the NDVI. NDVI calculation is shown in equation
$1:$

$$
N D V I=\frac{N I R-R E D}{N I R+R E D}
$$

where $\quad$ NIR $=$ pixel value near of infrared band

RED = pixel value of red color band

NDVI = resultant NDVI pixel value

Cropped images of these three bands are combined to form a false color composite image. Thus, in total, two images were formed for 2006 October and 2016 October, respectively. As the last step of the preprocessing, histogram of the first image is equalized to the second one. Resulting images are illustrated in Figure 3 and 4 with their corresponding time stamps.

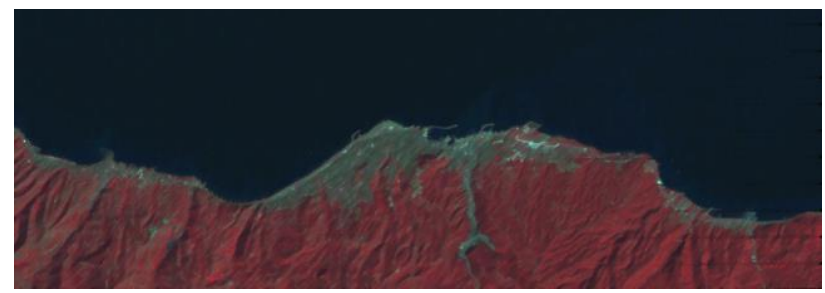

Figure 3. False color composite image - 06.10.2006

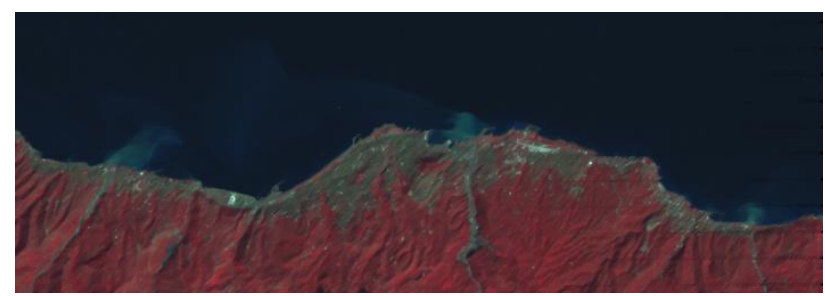

Figure 4. False color composite image - 01.10.2016

Images representing calculated NDVI are shown in Figure 5, Figure 6 . To verify the integrity of this technique, the resulting NDVI image, in which each pixel is in $\{-1,1\}$ interval, was first converted into a binary image using an accepted vegetation threshold value of 0.2 for this kind of analyses (FSNAU, 2017, USGS, 2015, Wikipedia, 2017). On the other hand, extreme negative values represent water, median values that are approximately equal to 0 represent bare soil. After applying such a conversion for both dates, the resultant binary images were compared to the binary ground truth images which are prepared using Photoshop CS5 lasso tool and MS Paint. The results are represented by the metrics presented in Table 1 . The overall accuracy for 2006 image prediction is 0.956 and 2016 image is 0.9707 .

\begin{tabular}{|l|l|l|}
\hline Statistics/Year & $\mathbf{2 0 0 6}$ & $\mathbf{2 0 1 6}$ \\
\hline TP & 107595 & 106437 \\
FP & 7489 & 3845 \\
TN & 228280 & 234618 \\
FN & 7987 & 6451 \\
Precision & 0.935 & 0.965 \\
Recall & 0.931 & 0.943 \\
fScore & 0.933 & 0.954 \\
Overall Accuracy & 0.9560 & 0.9707 \\
\hline
\end{tabular}

Table 1. Confusion matrix for images of years 2006 and 2009, listing their true/false $(\mathrm{T} / \mathrm{F})$ positive/negative $(\mathrm{P} / \mathrm{N})$ detected

pixel numbers, overall accuracy, precision and recall in detecting vegetation, and two fScore combining precision and recall values for each image. 
In addition to accuracy, precision and recall values for both images were also calculated. Recall metric represents the rate of the correctly identified vegetated regions and calculated as TP / $(\mathrm{TP}+\mathrm{FN})$. On the other hand, precision indicates what percentage of the regions found as vegetation are actually true with respect to ground truth and calculated as TP / (TP + FP). Using precision and recall, we can calculate an fScore value for prediction of vegetated regions, which is a score varies in $[0,1]$ range. The higher the score value, the better the result is. The formula for fScore is shown in equation 2 .

$$
\text { fScore }=2 \times \frac{(\text { Precision } \times \text { Recall })}{(\text { Precision }+ \text { Recall })}
$$

where Precision $=$ precision value for the case

Recall $=$ recall value for the case

$\mathrm{fScore}=$ resultant fScore value

For both images, it can be concluded that vegetated regions were detected accurately due to the high precision and recall values are obtained which indicates the robustness of the technique. Therefore, following analysis can be reliably done in order to identify the change in the vegetation ratio of the study area.

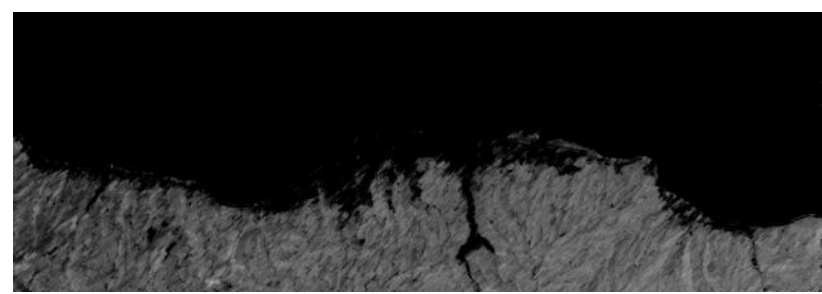

Figure 5. NDVI image - 06.10.2006

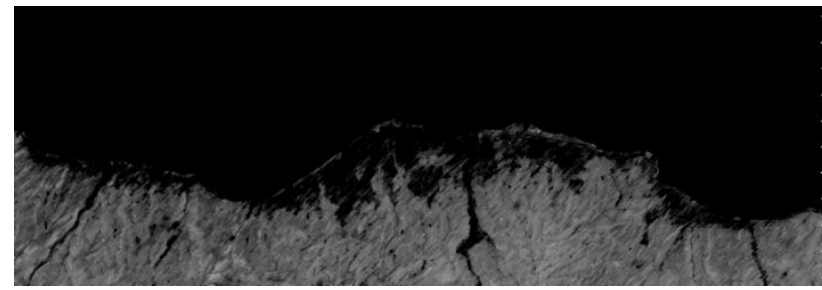

Figure 6. NDVI image - 01.10.2016

Vegetation ratio of the study area were also calculated using equation 3. Ratios are found as 0.328 and 0.314 for years 2006 and 2016 respectively.

$$
\text { Vegetation Ratio }=\frac{\text { Number of pixels with value }=1}{\text { Total number of pixels }}
$$

Examining the vegetation ratios, it can be said that there is a loss in vegetated regions in 2016. Therefore, as a final step the loss of the vegetation ratio within this interval was calculated, by accepting the first ratio value (corresponding to 2006 image) as the basis. Thus, the loss rate between 2006 and 2016 is $(0.328$ $-0.314) / 0.328=0.0427(\% 4.27)$.

Detected vegetation differences are illustrated in Figure 7, in which white areas represent vegetated regions in both years and black areas indicate non-vegetated regions. On the other hand, red regions represent vegetated areas of 2006 which are not present in 2016, and green areas are non-vegetated areas of 2006 which are detected as a vegetation region. However, the green areas around "Sögütlü River" are detected as a non-vegetation index in 2006 because of the contour that occurred due to shadows. This has to be related to the fact that these images were gathered in different times of the day. Considering this, total deforestation rate should be approximately $\% 5$.

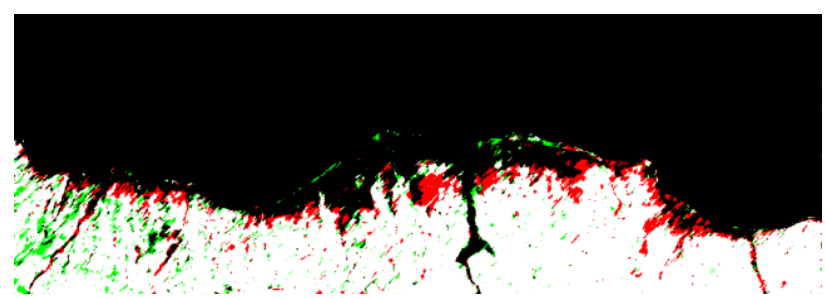

Figure 7. Vegetation change image between 2006 and 2016

A visual inspection was also performed with images from Google Timelapse (Google, 2017) and urbanization in Trabzon and neighboring towns that are shown in Figure 8 and 9. It is clear that urban areas grew between 2006 and 2016 and as a consequence, a loss in vegetated regions occurred which supports our result.

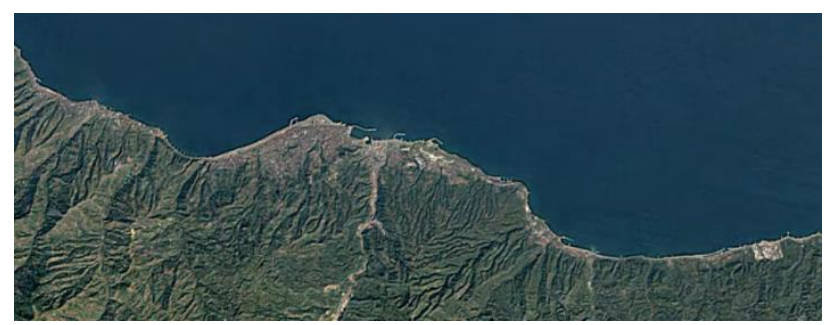

Figure 8. Google Timelapse Image of Trabzon (2006)

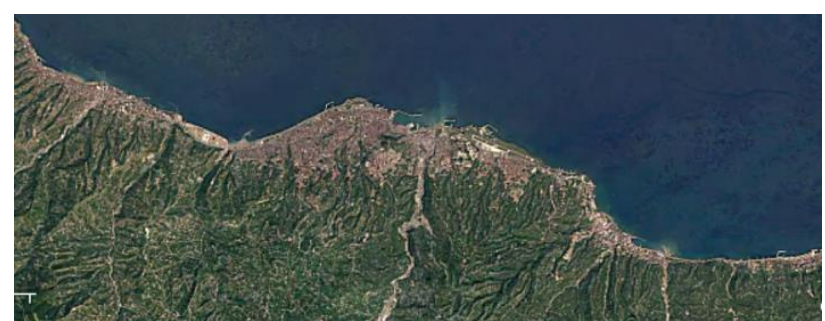

Figure 9. Google Timelapse Image of Trabzon (2016)

\section{RESULTS AND CONCLUSION}

In this study, we have examined the deforestation rate around Trabzon city center and surrounding towns, Akçaabat, Yalıncak, Yomra, Yeşilyaylı, and others. As a result \%4.27 total deforestation is calculated. One interesting result is the, aspect and timing of the taken satellite imagery may yield erroneous results. Yet, deforestation due to urbanization in these areas should be prevented. Various serious events, like fires, take place and rumors point out that these are intended to clean out forestry for building more hotels or other facilities (CNN Turk, 2017). Google Timelapse is very useful to observe the urbanization BOOM in different cities of the world. Further, how the global warming melts the ice can be observed at the tip of Greenland.

\section{REFERENCES}

CNN Turk, 2017. “Trabzon' da Orman Yangını”. http://www.cnnturk.com/video/turkiye/trabzonda-ormanyangini.

Csillik O., 2017. "Fast Segmentation and Classification of Very High Resolution Remote Sensing Data Using SLIC Superpixels", Remote Sensing 2017, 9, pp. 243. 
FSNAU, Food Security and Nutrition Analysis Unit of Somalia, 2017. "Understanding the Normalized Difference Vegetation Index (NDVI)", http://www.fsnau.org/downloads/Understanding_the_Normalize d_Vegetation_Index_NDVI.pdf.

GIS Geography, 2017. "Landsat Program: Satellite Imagery Data and Bands". http://gisgeography.com/landsat-programsatellite-imagery-bands/.

Google, 2017. "Google Earth Engine - Timelapse". https://earthendine.google.com/timelapse/.

Hollstein, A., 2016. "Ready-to-Use Methods for the Detection of Clouds, Cirrus, Snow, Shadow, Water and Clear Sky Pixels in Sentinel-2 MSI Images”. Remote Sensing. 2016, 8, pp. 666.

Machado, R.J., 1993. Monitoring the deforestation of the Amazon region with neural networks, IBM Rio Scientific Center, Proceedings of International Joint Conference on Neural Networks.

MathWorks, 2017. "imclose: Morphologically Close Image". https://www.mathworks.com/help/images/ref/imclose.html.

McCabe, M., 2017, "Gap-Filling of Landsat 7 Imagery Using the Direct Sampling Method”. Remote Sens. 2017, 9, 12.

Menaka, E., 2013. Change Detection in Deforestation using High Resolution Satellite Image with Haar Wavelet Transforms. In: Proceedings of 2013 International Conference on Green High Performance Computing March 14-15, 2013, India.

MSXLAB Forum, 2008. "Karadeniz Bölgesi' nde yetişen tarım ürünleri

https://www.msxlabs.org/forum/cevaplanmis/214561-

karadeniz-bolgesinde-yetisen-tarim-urunleri-nelerdir.html.

Pacheco, C.E., 2014. Identification and characterization of deforestation hot spots in Venezuela using MODIS satellite images, Acta Amazonica, VOL. 44(2) 2014: pp. 185 - 196.

U.S. Geographical Survey, 2015. "NDVI, the Foundation for Remote Sensing Phenology", https://phenology.cr.usgs.gov/ndvi_foundation.php.

U.S. Geographical Survey, 2017a. "Earth Explorer", https://earthexplorer.usgs.gov/.

U.S. Geographical Survey, 2017b. "Filling the Gaps for Display”. https://landsat.usgs.gov/filling-gaps-display.

U.S. Geographical Survey, 2017c. "Filling Gaps to Use in Scientific Analysis", https://landsat.usgs.gov/filling-gaps-usescientific-analysis.

U.S. Geographical Survey, 2017d. "What are the band designations for the Landsat satellites? https://landsat.usgs.gov/what-are-band-designations-landsatsatellites.

Wikipedia, 2017. "Normalized Difference Vegetation Index". https://en.wikipedia.org/wiki/Normalized_Difference_Vegetatio n_Index.

Zhang, C., 2007. Gaps fill of SLC off Landsat ETM+ Satellite Image Using a Geostatistical Approach. International Journal of Remote Sensing, 28:22, pp. 5103-5122.
Revised June 2017 